\title{
In Vitro Evaluation of Natural Keratin Based Hydrogel from Chicken Feather Waste for Controlled Drug Release
}

\author{
D. Sakthi Chandini ${ }^{1}$, M. Charulatha ${ }^{2}$, R. Legadevi $^{2}$ and S. Meignanalakshmi ${ }^{2 *}$ \\ ${ }^{1}$ Madha Engineering College, Chennai-69, Tamil Nadu, India \\ ${ }^{2}$ Department of Animal Biotechnology, Madras Veterinary College, TANUVAS, \\ Chennai-7, Tamil Nadu, India \\ *Corresponding author
}

\section{A B S T R A C T}

\section{Keywords}

Chicken Feathers, Keratin Hydrogel, Drug encapsulation.

Article Info

Accepted:

28 September 2017

Available Online:

10 October 2017
In the present study keratin was extracted from chicken feather using the reductants beta mercaptoethanol, urea and SDS. The extracted keratin was lyophilised and was used to prepare hydrogel. Prepared hydrogel was characterized using SEM and FTIR. The prepared keratin hydrogel was used for encapsulation of tetracycline. The percentage of drug encapsulation in keratin hydrogel was found to be $92.5 \%$. The in vitro release of tetracycline from keratin hydrogel was carried out in PBS for $24 \mathrm{hrs}$ and was found to be released in a slow and sustained manner.

\section{Introduction}

Keratin is a natural protein which has its source in animal hair, feathers, nails, hooves, beaks, claws and horns. The keratin protein is a stable protein which cannot be dissolved in any organic or inorganic solvents and it cannot be cleaved by protease enzymes such as trypsin or pepsin (Goddard and Leonor, 1935).

The stable structural morphology of the keratin protein makes it highly a versatile protein. The keratin protein can be manipulated into any bioproduct including films, scaffolds, sponges, nanoparticles and hydrogel. They have the ability to selfassemble themselves to form a stable structure or morphology.
Keratin protein can be extracted from many source, one of the abundant source is chicken feathers. The chicken feathers possess $91 \%$ of keratin protein (Khosha and Ullah, 2013). The chicken feather discards are generated into the environment in tones. The chicken feathers are dumped in the lands cause environmental issues such as production of methane or greenhouse gases and pollution of the ground water sources (Staron et al., 2014; Evans and Vance, 2007). It needs some effective management system.

Hydrogels are three-dimensional network structures or polymeric structure which can absorb and retain significant amount of water. Usually it is made of natural or synthetic 
polymers as a structural backbone (Syed et al., 2011). These structures are a group of hydrophilic domains together bonded or cross linked in an aqueous environment. Because of these properties, hydrogel are used as a biomaterial in the field of biomedical engineering and also used in the field of tissue engineering, regenerative medicine, controlled drug delivery, biomaterial science, and polymer system studies (Enas, 2015).

The researchers in the biomedical field are in search of a suitable drug delivery system which could able to deliver the drug in a controlled manner and also reach the targeted site of infection more specifically.

Keratin proteins are self-assembling proteins and they can polymerize into a complex three dimensional structures like hydrogel and cell scaffold. Hence the present study focuses on the preparation of keratin hydrogel from chicken feather and to encapsulate the hydrogel with drug for controlled drug release studies.

\section{Materials and Methods}

\section{Pre-treatment of feathers}

Chicken feathers were collected from poultry shop, Vepery, Chennai. The chicken feathers were cleaned well with water and detergent for the removal of blood stain and other impurities. Cleaned feathers were washed again with water and $70 \%$ ethanol. The cleaned feathers were dried under sunlight. After drying the feathers were cut into small pieces and stored for further use.

\section{Extraction and estimation of keratin}

About $0.6 \mathrm{~g}$ of pre-treated feathers was added into $25 \mathrm{~mL}$ of aqueous solution containing $8 \mathrm{M}$ urea, $1.66 \mathrm{M}$ 2Mercaptoethanol and $0.17 \mathrm{M}$ Sodium dodecyl sulphate (Akira Tachibana et al., 2002; 2005) and kept at $60^{\circ} \mathrm{C}$ water bath for about 4 hrs. After 4 hrs of incubation, the mixture was allowed to cool and filtered using No 1 Whatman filter paper (GE health care UK limited) and was lyophilized. The keratin extracted from chicken feather was estimated by Lowry's method (1951).

\section{Preparation of keratin hydrogel}

$50 \mu \mathrm{L}$ of $1 \mathrm{X}$ phosphate buffer was added to $40 \mathrm{mg}$ of keratin gel powder. Hydrogel formation was observed by the addition of phosphate buffer. Obtained keratin hydrogel was further characterized and used for drug encapsulation.

\section{Characterization of keratin hydrogel}

Keratin hydrogel was characterized using Scanning Electron Microscope (SEM) and FTIR.

Encapsulation of hydrogel with tetracycline

$0.37 \mathrm{mg}$ of tetracycline suspended in PBS was added to $40 \mathrm{mg}$ of keratin gel powder. The hydrogel was allowed to equilibrate in tetracycline solution for 24 hours. After 24 hours the drug loaded hydrogel was used for drug release studies (Das Manali and Nirada Devi, 2015; Gabriela Buhus et al., 2009).

The following formula is used to find the percentage of drug loaded

$\mathrm{DL}=\frac{\mathrm{W} 1-\mathrm{W} 2}{\mathrm{~W} 2} \times 100$

W1-Weight of the drug loaded hydrogel

W2-Weight of the dry hydrogel

DL-Percentage of drug loaded

\section{In vitro drug release studies}

The drug loaded hydrogel was immersed separately in $10 \mathrm{~mL}$ of distilled water. At 
regular time intervals the medium was taken out and the absorbance was read at 420nm (Das Manali and Nirada Devi, 2015; Gabriela Buhus et al., 2009). The absorbance values were used to determine the concentration of drug released from keratin hydrogel. The standard curve was plotted using the known concentration of the tetracycline and their respective optical density.

\section{Results and Discussion}

\section{Estimation of keratin hydrogel}

The amount of keratin extracted from chicken feather was found to be $0.58 \mathrm{mg} / \mathrm{ml}$.

\section{Characterization of keratin hydrogel by} scanning electron microscope (SEM)

The keratin hydrogel prepared was characterised using SEM. The SEM images (Fig 1) showed the structure and morphology of the hydrogel. The images revealed the internal structure of the hydrogel. SEM revealed the presence of pores in the hydrogel and the cross linking of the keratin. Sizes of pores were uniform. The sharp peak like structures was observed in the keratin structures, which were self-assembled and crosslinked to form the pores.

\section{FTIR spectrum of keratin extract}

The keratin extract obtained showed peaks (Fig 2) between $1701 \mathrm{~cm}^{-1}, 1699 \mathrm{~cm}^{-1}$ to $1600 \mathrm{~cm}^{-1}$ and it showed another peaks between $1560 \mathrm{~cm}^{-1}$ to $1500 \mathrm{~cm}^{-1}$, it Proved the presence of both amide 1 and 2 group. There were four peaks between $1656 \mathrm{~cm}^{-1}$ to $1650 \mathrm{~cm}^{-1}$ and four peaks between $1550 \mathrm{~cm}^{-1}$ to $1541 \mathrm{~cm}^{-1}$ which proved the presence of alpha helical structure. The keratin extract also showed peaks between $1635 \mathrm{~cm}^{-1}$ to $1614 \mathrm{~cm}^{-1}$ and peaks between $1535 \mathrm{~cm}^{-1}$ to $1521 \mathrm{~cm}^{-1}$. These peaks confirmed the presence of beta sheets in it. The number of peaks observed was more in the beta conformation range. Therefore the conformation contains larger distribution of beta sheets in its protein morphology.

\section{FTIR of keratin hydrogel}

The peaks obtained in the keratin gel were matched well with the keratin extract spectrum. The slight changes in the keratin gel spectrum was observed due to the cross linking of the disulphide bonds or reformation of bonds to form a polymeric hydrogel.

The hydrogel powder showed various peaks (Fig 3), which confirmed the predominant presence of beta sheets and less distribution of alpha helix. The peaks were quite elevated in the keratin extract between the region $2265 \mathrm{~cm}^{-1}$ to $125 \mathrm{~cm}^{-1}$. The keratin gel didn't show any sharp peak as it was in the keratin extract in that particular range. The peaks between the range $3288 \mathrm{~cm}^{-1}$ to $3309 \mathrm{~cm}^{-1}$ proved the presence of the hydrogen bonded amine group. The high frequency of hydrogen bond and nitrogen group proved that the structure was more folded. Hence the more folded structures in keratin hydrogel were obtained. The comparison between keratin hydrogel and keratin extract proved that the formed product was keratin hydrogel.

\section{Drug encapsulation}

The percentage of drug encapsulated in keratin hydrogel was found to be $92.5 \%$.

\section{In-vitro drug release studies}

From the standard graph of the tetracycline, the concentrations of the drug released at different time interval was determined.

The concentration of the drug released per hour was very minimal and the release was 
carried out in a controlled manner. The drug release was constant throughout and hence this hydrogel was proved to be an efficient drug delivery system. The constant change in concentration of drug released is shown in fig 4.

Fig.1A, B, C, D and E SEM images of keratin hydrogel
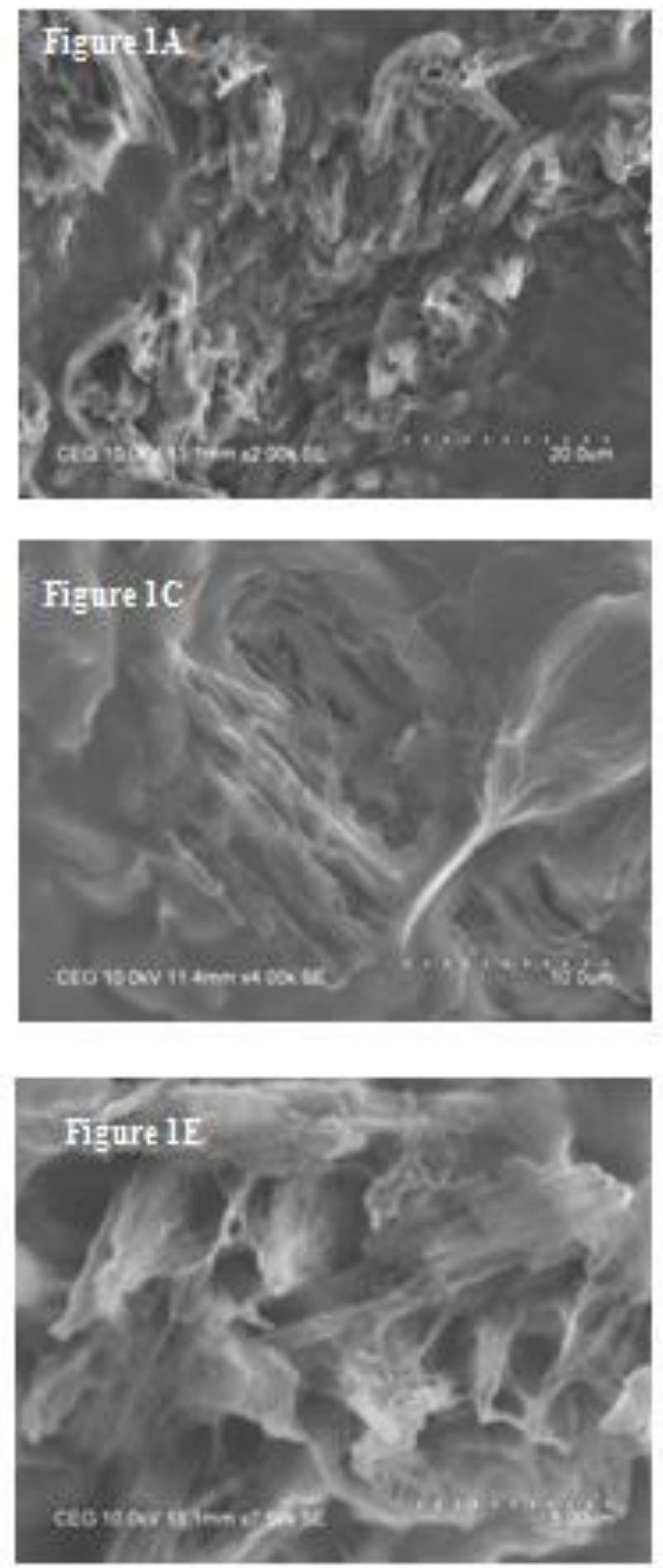
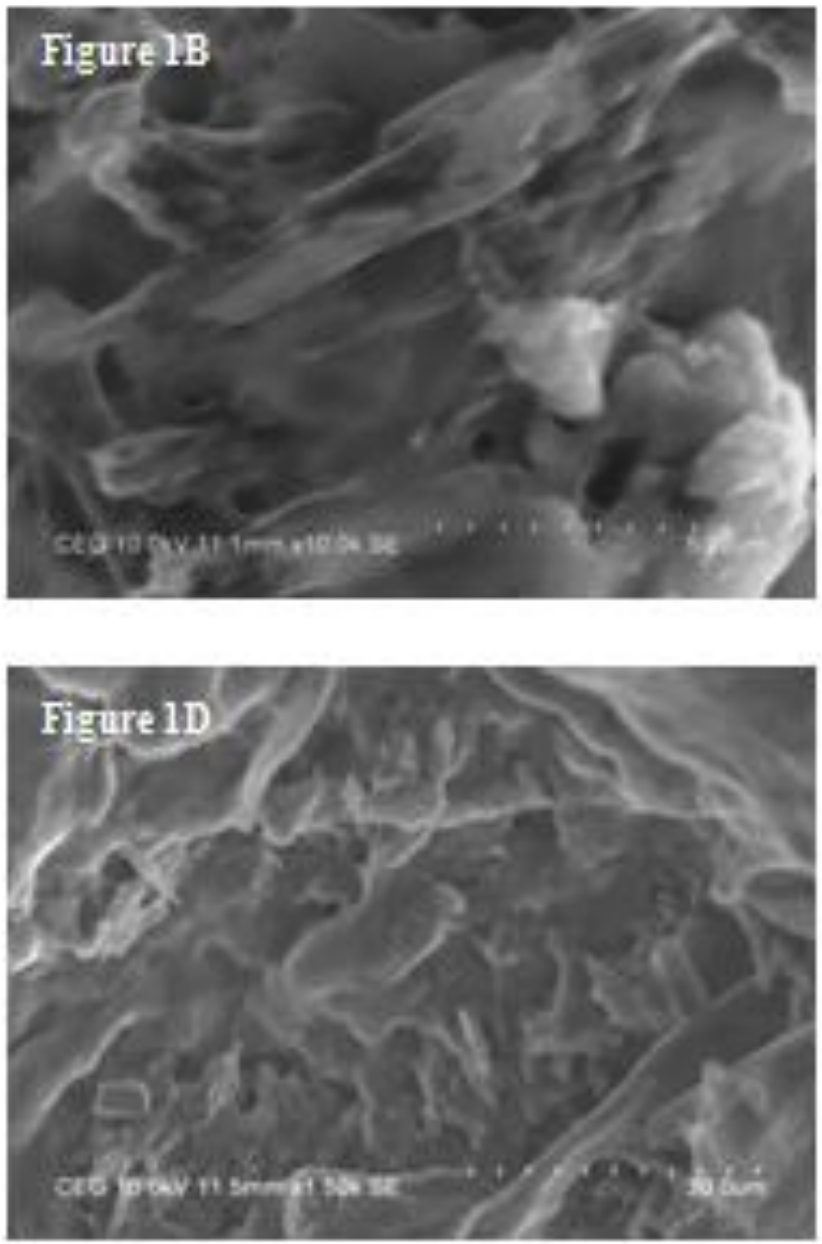
Fig.2 FTIR spectrum of keratin hydrogel
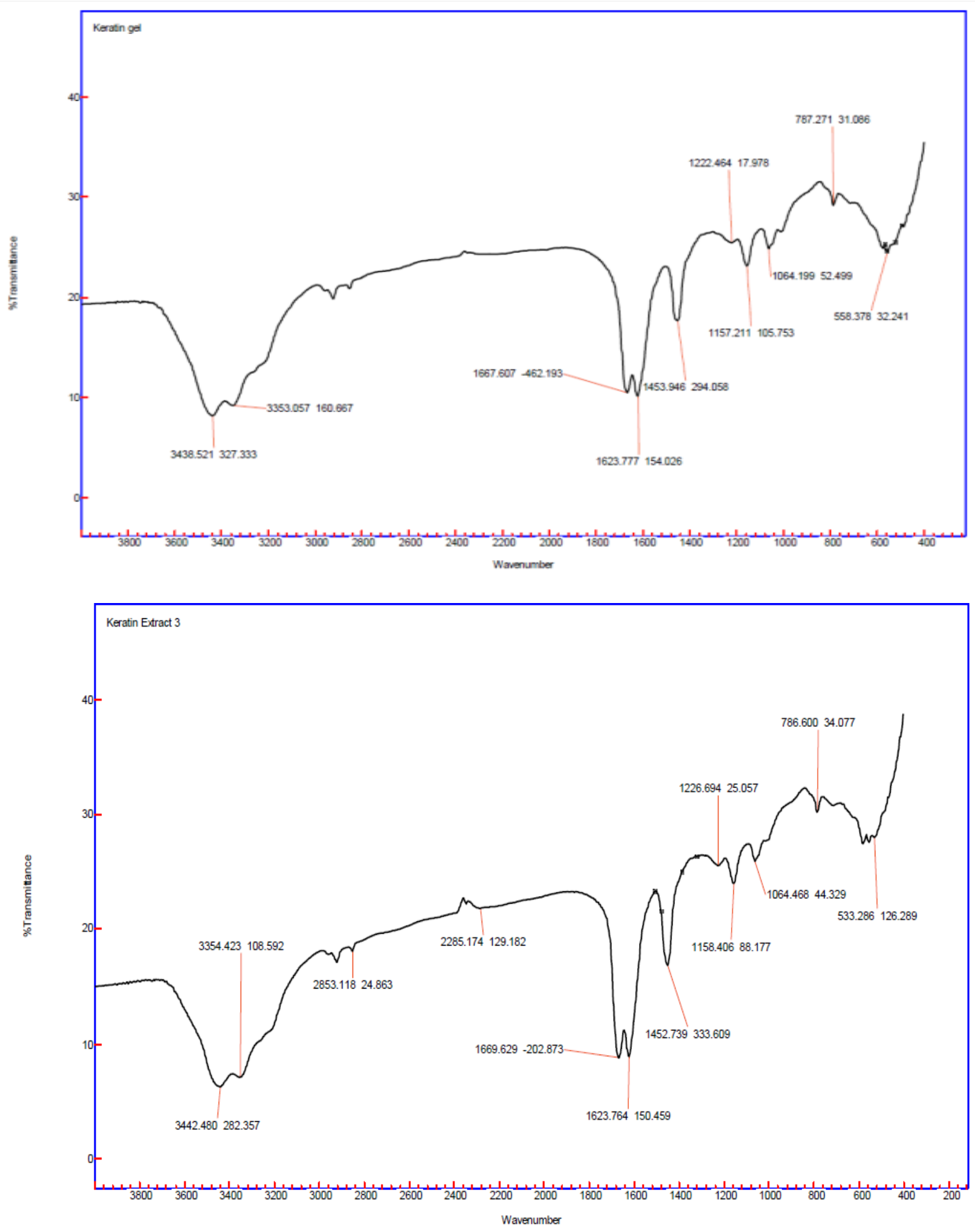
Fig.3 In vitro release of tetracycline from keratin hydrogel

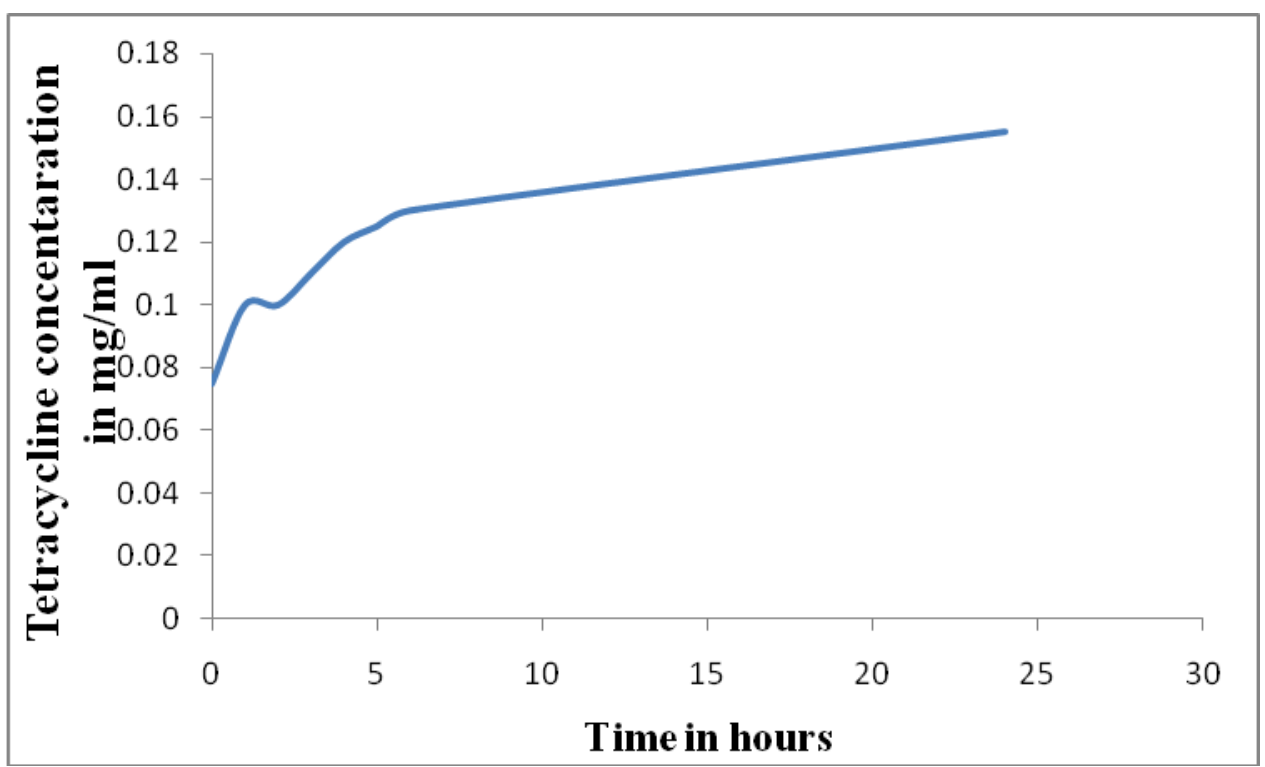

Here in our study keratin protein is used for the hydrogel preparation. The keratin obtained is more stable and can remain stable in extreme $\mathrm{pH}$. It has the ability to recrosslink the cysteine groups by oxidative coupling. The biomaterials formed from keratin are stable and can survive for months (Silva et al., 2014).

The keratin protein is extracted from the source using different sulfhydryl reductants. The sulfhydryl reductants like Dithiotreitol, tris (2-carboxyethyl) phosphine are reported by Elise Burmeister Getz et al., (1999). The keratin from chicken feathers can be extracted by employing reducing agents such as potassium cyanide, sodium sulphide and ammonium thioglycolate. Arun Gupta et al., (2012) has compared the keratin extracted from these three reducing agents to determine the efficient reducing agent that could possibly yield a high amount of keratin protein from chicken feathers.

However the most widely used reductant is beta mercaptoethanol. Mercaptoethanol effectively breaks disulphide bonds in protein and inhibits the oxidation of free sulfhydryl residues thereby maintaining the protein activity (Elise Burmeister Getz et al., 1999). In this study three reducing agents like beta mercaptoethanol, urea and sodium dodecyl sulphate were used in extraction for the effective yield (Akira Tachibana et al., 2002; 2005). The Sodium Dodecyl Sulphate is an anionic surfactant that can able to break the non-covalent bonds and protein linkages. Urea is also considered as protein denaturant that reduces the non-covalent bonds (Elise Burmeister Getz et al., 1999).

Akira Tachibana et al., has reported this method of reduction for reducing wool. However in the present study, these reducing agents were used for chicken feathers (Akira Tachibana et al., 2002; 2005).

The three major types of preparation of hydrogel are Chemical crosslinking, Physical crosslinking and radiation crosslinking (Syed et al., 2011). So far the keratin hydrogel preparation is done by all the three methods. Cardamone et al., (2013) prepared a wool based keratin hydrogel by oxidation and reduction hydrolysis method where a number of oxidising and reducing agents are used to 
prepare a keratin hydrogel. Mira Park et al., (2013; 2015) has prepared both wool based and human hair based keratin hydrogel by irradiating electron beam at different ranges and also studied the stability of the protein gel. Though the hydrogel is stable the method of preparation involves electron beam for the crosslinking of the gel.

Rabiatul Adawiyah Binti Zaukifli (2012) has prepared keratin hydrogel by adding poly vinyl chloride to the extract using freeze thaw method. Silva et al., (2014) has prepared the keratin gel using physical pressures such as pressure driven extrusion and sonochemical method. Sujuan Pan et al., (2015) has designed a hydrogel by graft polymerisation technique using methacrylic acid, $\mathrm{N} \mathrm{N}$ bis acralamide and agar.

However in this study, the gel like structure is formed during the extraction process itself. The disulphide bridges were broken and formed a gel like structure. The gel structures or hydrogel was lyophilized into powders and they are restructured into a gel by adding phosphate buffer saline. This method of hydrogel preparation is simple, cost effective and efficient. The other authors have also used Phosphate buffer saline for hydrogel preparation but the method of extraction is by partial oxidation and reduction and the source is from human hair (Lauren et al., 2014).

The keratin hydrogel prepared by using chicken feather waste was found to be suitable for encapsulation of drugs and also to release drug in a controlled manner.

\section{References}

Akira Tachibana, Sumika Kaneko, Toshizumi Tanabe and Kiyoshi Yamauchi. 2005. Rapid fabrication of keratinhydroxyapatite hybrid sponges toward osteoblast cultivation and differentiation. Biomaterials. 26: 297302.

Akira Tachibana, Yasunari Furuta, Hieyuki Takeshima, Toshizumi Tanabe and Kiyoshi Yamauchi. 2002. Fabrication of wool keratin sponge scaffolds for long term cell cultivation. Journal of biotechnology. 93, 165-170.

Arun Gupta, Ramanan Perumal, Roslin Bin Mohd Yunus and Nuruldiyanah Binti Kamarudin. 2012. Extraction of keratin from chicken feather. University of Malaysia Pahang, Gambang Campus, 25100, Kuantan Phang, Malaysia. Journal of chemistry and chemical engineering. 6(8): 732-737.

Cardamone, Jeanette, M., Tunick, Michae, H. and Charles Onwulata. 2013. Keratin Sponge/Hydrogel: 1.Fabrication and Characterization. Textile research Journal. 83 (7): 661-670.

Das Manali and Nirada Devi. 2015. Preparation, characterisation, water sorption and drug release study of 2 poly acralamide-2-methylpropane sulfonic acid (AMPS) based hydrogel. International journal of research in chemistry and environment. 5(1): 69-75.

Elise Burmeister Getz, Ming Xiao, Tania Chakrabarthy, Roger Cooke and Paul, R. Selvin. 1999. A comparison between the sulfhydryl reductants Tris(2carboxyethyl) phosphine and dithiothreitol for use in protein biochemistry. Analytical Biochemistry. 273, 73-80.

Enas, M. Ahmed. 2015. Hydrogel: Preparation, characterization, and applications: A review, Journal of Advanced Research. 6(2): 105-121.

Evans, Michael R. and Vance Leisha. 2007. Physical properties of processed poultry feather fiber-containing greenhouse root substrates. Hortechnology. 17 (3): 301304. 
Gabriela Buhus, Catalina Peptu, Marcel Popa and Jacques Desbrieres. 2009. Controlled release of water soluble antibiotics by carboxymethylcelluloseand gelatin-based hydrogels crosslinked with epichlorohydrin. Cellulose Chemical Technology. 43 (4-6): 141151.

Goddard, D.R. and Leonor M. 1935. Derivatives of keratin. J. biol.chem. $112,361-371$.

Khosha, A and Ullah, A. 2013. Sustainable role of keratin biopolymer in green chemistry: A Review. Journal of food processing and beverages. 1(1): 1-8.

Lauren, A. Pace, Johannes, F. Plate, Sandeep Mannava, Jonathan, C. Barnwell, Andrew Koman L., Zhongyu Li, Thomas, L. Smith and Mark Van Dyke. 2014. A human hair keratin hydrogel scaffold enhances median nerve regeneration in nonhuman primates: An electrophysiological and histological study. Tissue engineering, part A. 20(3 and 4): 507-517.

Lowry, O.H., Nira, J. Rosebrough, Lewis Farr, A. and Rose, J. Randall. 1951. Protein measurement with the folin phenol reagent. The Journal of Biological Chemistry. 193(1): 265-275.

Mira Park, Byoung-Suhk Kim, Hye Kyoung Shin, Soo-Jin Park and Hak-Yong Kim. 2013. Preparation and characterization of keratin-based biocomposite hydrogels prepared by electron beam irradiation. Material science and engineering C. 33, 5051-5057
Mira Park, Hye Kyoung Shin, Byoung-Suhk Kim, Myung Jin Kim, In-Shik Kim, Byung-Yong Park and Hak-Yong Kim. 2015. Effect of discarded keratin-based biocomposite hydrogels on the wound healing process in vivo. Material science and engineering C. 55, 88-94

Rabiatul Adawiyah Binti Zaukifli. 2012. Thesis, formulation of wound healing hydrogel from keratin protein, faculty of chemical \& natural resources engineering, University Malaysia Pahang.

Silva R., Ben Fabry and Aldo, R Boccaccini. 2014. Fibrous protein based hydrogels for cell encapsulation. BiomaterialsElsevier. 35, 6727-6738.

Staron, P., Banach, M., Kowalski, Z. and Staron, A. 2014. Hydrolysis of keratin materials derived from poultry industry. Proceedings of ECOpole. 8(2): 443-448.

Sujuan Pan, Xiaochun Yin, Yu-

Feng He, Yubing Xiong and RongMin Wang. 2015. Influence of Preparation Conditions on the Properties of Keratin-Based Polymer Hydrogel. Arabian Journal for Science \& Engineering (Springer Science \& Business Media B.V.). 40(10): 28532859.

Syed, K.H. Gulrez, Saphwan Al-Assaf and Glyn O Phillips. 2011. Hydrogels: methods of preparation, characterisation and applications. Intech open. Glyn O Phillips Hydrocolloids research centre Glyndwr University, Wrexham United Kingdom.

\section{How to cite this article:}

Sakthi Chandini, D., M. Charulatha, R. Legadevi and Meignanalakshmi, S. 2017. In Vitro Evaluation of Natural Keratin Based Hydrogel from Chicken Feather Waste for Controlled Drug Release. Int.J.Curr.Microbiol.App.Sci. 6(10): 3488-3495. doi: https://doi.org/10.20546/ijcmas.2017.610.411 